\title{
Late onset oral treatment with tranilast following large myocardial infarction has no beneficial effects on cardiac remodeling and mortality in rats
}

\author{
STEFAN BETGE $^{1}$, CHRISTIAN KUNZ ${ }^{2}$, HANS FIGULLA ${ }^{1}$ and CHRISTIAN JUNG ${ }^{1}$ \\ ${ }^{1}$ Department of Internal Medicine I, Division of Cardiology, Friedrich Schiller University, Jena, Thuringia 07740; \\ ${ }^{2}$ Department of General, Abdominal and Thoracic Surgery, City Hospital Dessau, \\ Dessau-RoBlau, Saxony-Anhalt 06847, Germany
}

Received February 24, 2014; Accepted August 20, 2014

DOI: $10.3892 /$ etm.2014.2003

\begin{abstract}
Tranilast (Tra) reduces intracardiac interstitial fibrosis in the animal models of hypertensive heart failure and diabetic cardiomyopathy by inhibiting cardiac fibroblasts. The present study examined whether Tra has long-term effects on the cardiac remodeling in the remote area of the left ventricle (LV) following myocardial infarction (MI) in the rat. Treatment with $\operatorname{Tra}(\mathrm{n}=40 ; 150 \mathrm{mg} / \mathrm{kg}$ twice daily) or placebo (Plac, $\mathrm{n}=36$ ) was started at day 28 after induction of a large MI or sham-operation $(\mathrm{ShO}, \mathrm{n}=18)$ in female Lewis rats. Collagen content was determined using high-performance liquid chromatography. Large MI led to a significant hypertrophy of the two ventricles, a severe dilatation of the LV and a shift of the chamber stiffness variables in the pressure volume curves. The six-month survival rates were Tra, 62.5\%; Plac, 75\%; and $\mathrm{ShO}, 100 \%$. No significant difference was identified between Tra and Plac regarding survival rate and collagen content. Treatment with the anti-inflammatory and antifibrotic drug, Tra, started four weeks after the induction of a large MI in the rat, did not attenuate or positively influence remodeling in chronic ischemic heart failure and survival. Further studies are required to explore the effects of Tra on cardiac myocytes post-MI in more detail.
\end{abstract}

\section{Introduction}

Tranilast was introduced as an anti-atopic agent forty years ago (1). Several studies and clinical reports have described anti-inflammatory and antifibrotic effects in the following years and tranilast was used for the treatment of dermatological

Correspondence to: Dr Christian Jung, Friedrich Schiller University, Department of Internal Medicine I, 101 Erlanger Allee, Jena 07740, Germany

E-mail: Christian.Jung@med.uni-jena.de

Key words: ischemic heart failure, antifibrotic, transforming growth factor, fibroblast, collagen content disorders, such as scleroderma, cheilitis granulomatosa, granuloma anulare or sarcoidosis (2,3). In cell culture experiments, it has been shown that tranilast inhibits collagen synthesis in fibroblasts, partially as a direct effect on the protein expression and partially via inhibition of the stimulating effect of transforming growth factor $\beta 1$ (TGF- $\beta 1$ ) on the collagen synthesis of fibroblasts $(4,5)$. Tranilast prevented intraperitoneal adhesions in an animal model (6) and it has been used for the prevention of hypertrophic scar development following sternotomy in children (7).

Tranilast inhibited the collagen synthesis and proliferation of vascular smooth muscle cells in cell culture experiments by interacting with the receptors for the platelet-derived growth factor, TGF- $\beta$, and angiotensin II (8). These effects could be translated in a significant reduction of the intimal hyperplasia subsequent to treatment with tranilast in different animal models following arterial injury (9), into reduced restenosis rates subsequent to percutaneous coronary angioplasty of de novo or restenotic lesions in smaller human studies $(10,11)$, but not in the large Prevention of REStenosis with Tranilast and its Outcomes trial $(11,12)$. In addition, tranilast showed positive effects on intracardiac inflammatory processes positively influencing cardiac remodeling in several animal models $(13,14)$ : Tranilast administered once daily for four weeks led to a decrease of the left ventricular hypertrophy and ameliorated the perivascular and interstitial intracardiac fibrosis in spontaneously hypertensive rats. The left ventricular end diastolic pressure or chamber stiffness constants were not affected (13). Similar results were obtained in the animal model of uni-nephrectomized deoxycorticosterone acetate (DOCA)/salt hypertensive rats. Tranilast administered for 28 days attenuated the intracardiac perivascular and interstitial fibrosis, in parallel with an inhibition of TGF- $\beta_{1}$ expression and a suppression of cardiac mRNA levels of different cytokines (14).

In contrast to these animal models the processes leading to the cardiac remodeling following myocardial infarction (MI) do not involve the entire circumference of the left ventricle (LV). Cardiac remodeling following large MI can be interpreted as a response of the remote non-infarcted section of the LV to changes in ventricular geometry. However, the 
Table I. Age and body weight of the rats at the time of randomization.

\begin{tabular}{lrrrr}
\hline Characteristic & Plac $(\mathrm{n}=75)$ & Tra $(\mathrm{n}=75)$ & ShO $(\mathrm{n}=20)$ & P-value \\
\hline Age, weeks & $24.5 \pm 0.9$ & $26.2 \pm 0.5$ & $25.5 \pm 0.8$ & 0.20 \\
Body weight, $\mathrm{2}$ & $219.8 \pm 2.5$ & $215.1 \pm 3.1$ & $223.8 \pm 3.6$ & 0.26 \\
\hline
\end{tabular}

Data are presented as the mean \pm standard error of the mean. Groups: Plac, large myocardial infarction/placebo; Tra, large myocardial infarction/tranilast; ShO, sham-operation/placebo.

key features of cardiac remodeling are the same, involving myocyte hypertrophy, apoptosis and interstitial fibrosis. Several data show that the inflammatory processes in the ischemic area, as well as in the remote non-infarcted area of the LV following MI, are involved in the different phases. TGF- $\beta$ is believed to play a central role in each of these phases. TGF- $\beta$ promotes extracellular matrix protein expression by fibroblasts and inhibits matrix degradation via several mechanisms (15).

In experimental models of MI the expression of different isoforms of TGF- $\beta$ was found to be upregulated in different phases post-infarction. TGF- $\beta_{1}$ and $-\beta_{2}$ are induced in the early phase, whereas TGF- $\beta_{3}$ shows delayed and prolonged upregulation (16). The process of cardiac remodeling outside the infarcted area of the LV starts within hours following the infarction and continues to progress over weeks or months (17). An animal study with mice revealed that the blockade of TGF- $\beta$-signaling by overexpression of the extracellular domain of the TGF- $\beta$ type II receptor during the early phase following MI resulted in left ventricular dilatation and increased early mortality. By contrast, blockade of the TGF- $\beta$ signaling in the later phase following MI prevented the LV dilatation and the reduction of the contractile function, as well as myocyte hypertrophy and interstitial fibrosis (18). Recently, the study by See et al (19) reported on early and late administration of tranilast following MI (early, between $24 \mathrm{~h}$ and seven days post-MI; late, 7-28 days post-MI). The study revealed that tranilast inhibited myocardial TGF $\beta_{1}$ expression, fibrosis in rat post-MI and collagen production in cardiac fibroblasts. However, tranilast intervention from $24 \mathrm{~h}$ post-MI exacerbated infarct expansion, delaying the commencement of treatment to seven days post-MI impeded LV remodeling. Therefore, the aim of the present study was to investigate extremely late tranilast administration (starting at day 28) and its effect on cardiac remodeling and the six-month mortality rate in an experimental model of chronic ischemic heart failure following a large MI in the rat.

\section{Materials and methods}

Animal model and study groups. Studies were performed on 268 female Lewis rats, inbred and raised in the Institute for Animal Experiments of the Friedrich Schiller University (Jena, Germany). The studies were approved by the Ethics Committee of the Friedrich Schiller University. The investigation conforms to the Guide for the Care and Use of Laboratory Animals published by the National Institutes of Health $\mathrm{NIH}$; publication no. 85-23, revised 1996) and to the German law on the protection of animals.
The study was designed in an intention-to-treat manner. During the randomization process the animals were designated to the different surgical/treatment groups (MI/tranilast, $\mathrm{MI} /$ placebo or sham-operation (ShO)/placebo), as well as to the different analysis groups (collagen content via high-performance liquid chromatography (HPLC) and resting pressure-volume-curve/histological studies) prior to surgery. For the induction of an MI, the proximal left anterior descending coronary artery was ligated via left lateral thoracotomy following tracheotomy for controlled ventilation (20). In the sham-operated animals, the suture only was loosely tied. Ribs, muscles and skin were closed in separate layers. The animals were housed for six months, four of each in one polyethylene cage, with a maintained $12 \mathrm{~h}$ light/dark cycle. Animals had free access to standard food and water ad libitum.

Due to a high mortality rate of $39.3 \%$ (97 animals) following ligation of the proximal left anterior descending coronary artery and $4.8 \%$ (one animal) following the $\mathrm{ShO}$ in the first $48 \mathrm{~h}$ after surgery, a total number of 268 animals were randomized and the surgery performed according to the protocol until the intended group sizes of $n=75$ in each group following $\mathrm{MI}$ and $\mathrm{n}=20$ following the ShO were reached. No difference was identified between the groups concerning body weight or age (Table I).

Drug administration. The administration of the study drug or placebo was performed at days 28-182 post-operation by gavage. The scar formation following MI was completed, so that the treatment with tranilast did not have an effect on the scar formation in the infarcted area of the LV. Tranilast was administered at a daily dose of $300 \mathrm{mg} / \mathrm{kg}$ in two doses, dispersed in a solution of Tylose $\mathrm{H} 300$, which served as the placebo as well.

Preparation. The rats that succumbed during the study period were autopsied and the hearts and lungs were excised. The rats surviving the experimental period were sacrificed by decapitation after 182 days. Hearts were perfused in situ with ice-cold heparinized physiological saline following cannulation of the proximal aorta and its ligation distal. The hearts were stopped in diastole by additional flushing with $7.45 \%$ potassium chloride. Transmural MI was clearly visible as a thin scar tissue with aneurysmatic bulging. Only rats with a transmural MI reaching from the base of the LV to its apex and including more than one-third of its circumference were included into further analysis. The resting pressure-volume curve analyses were performed in all the LVs, and the hearts 
were subsequently prepared either for chromatographical or histological determination of the collagen content.

Resting pressure-volume curve. A pressure-volume-curve of the resting isolated LV was performed in all the probes. The tip of a double-lumen catheter (polyethylene tube, innerlumen $0.5 \mathrm{~mm}$ inside polyethylene tube, innerlumen $2.0 \mathrm{~mm}$ ) was placed in the LV and ligated in the atrioventricular groove. The catheter was connected to a Statham pressure transducer to record a pressure-volume curve (infusion of $0.9 \%$ saline, $15 \mathrm{ml} / \mathrm{h}, \leq 30 \mathrm{mmHg}$ ) (21). For each heart, two measurements were performed within $10 \mathrm{~min}$ after cardiac arrest. Pressure and volume were recorded every second. These data were used for an automated regression curve (best-fit curve) analysis. At pressure ranges of 0-3 $\mathrm{mmHg}$, curves were generated following a linear model and were expressed as $y=a x+b$. The chamber stiffness is described by the slope of the curve, ' $a$ '. At pressure ranges of 3-10 and 10-30 $\mathrm{mmHg}$, exponential curves had to be constructed as regression curves to fit the data. These curves can be described as $y=c x d$. Subsequent to calculating the logarithm, $\log (y)$ can be expressed as a linear function of ' $d$ '. In the exponential model the chamber stiffness is described by ' $d$ '. To determine the ventricular dilatation, the infused volumes at 5, 10 and $15 \mathrm{mmHg}$ were measured for each heart.

Collagen content. The collagen content in the non-infarcted area of the LV was assessed using two different methods. In 30 animals, the hydroxyproline content of the probes of the non-infarcted inferior left ventricular wall was measured by HPLC. Two probes, each weighing 100-150 mg, were taken from each heart. Hydroxyproline is solely found in collagen, constituting a fraction of $13.4 \%$. Subsequent to homogenization in phosphate buffer and lyophilisation, the probes were hydrolyzed for $24 \mathrm{~h}$ at $114^{\circ} \mathrm{C}$ in $6 \mathrm{M} \mathrm{HCl}$. Hydroxyproline bound to an ion-exchanger (Dowex W-X8, Sigma-Aldrich, St. Louis, MO, USA) in phosphate-citric-acid-buffer ( $\mathrm{pH}$ 5.0), and was eluted from the ion-exchanger by heating up the probes to $115^{\circ} \mathrm{C}$ for $16 \mathrm{~h}$ and washing them with acetate-citrate-citric acid-buffer (pH 6.0). Aliquots were diluted in $20 \%$ acetate-buffer in methanol. The content of hydroxyproline in the probes was analyzed by HPLC with 7-chloro-4-nitrobenzofurazan as the fluorescent-labeling reagent. The results were expressed as nmol per probe and subsequently converted into microgram collagen per milligram dry weight of cardiac tissue.

The collagen content of the non-infarcted section of the LV was measured by planimetry of cryosections in 38 animals. The LVs were cut into four transversal cross-sectional slices of equal thickness and shock frozen in liquid nitrogen. Cryostat sections $(7-\mu \mathrm{m})$ were cut from each probe and stained with Masson's trichrome stain. Five optical fields (magnification, $\mathrm{x} 40$ ) in the non-infarcted section of each cryostat section were analyzed. These five fields were dispersed across the slice circumferences, but no field with tissue defects due to the cutting process or further preparation, nor an optical field with large vessels respectively perivascular collagen were analyzed. The blue-stained collagen could be clearly distinguished from cardiac myocytes (with red-stained myofibrils and brown nuclei) or defects. This differentiation was performed automatically using an individualized macro for the NIH-Scion Image ${ }^{\circledR}$ program (Scion Corp., Walkersville,

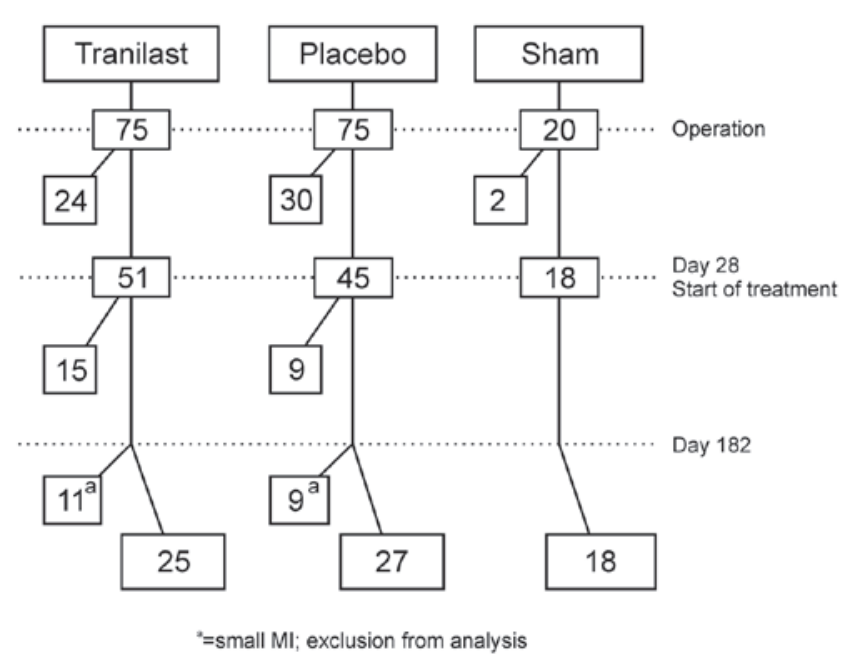

Figure 1. Flow diagram of the experimental settings. Branches to the left describe the mortality following the surgery and during the treatment period, and the exclusions due to small MI after day 182. MI, myocardial infarction.

MD, USA). The averaged gray scale values of all three colour channels were subtracted from the inverted gray scale values of the blue channel. A cut-off point was set to differentiate the blue staining. Calculation was performed as percentage of area using the pixel-based program. The hearts of these animals, which succumbed during the study period, were analyzed via histology.

Infarct size and thickness of the scar. The infarct size was measured as the percentage of the inner and outer diameter in the cryostat sections of each of the four transversal cross-sectional slices (22). The thickness of the scar was measured three times in each of the cryostat sections.

Statistics. All the values are expressed as mean \pm standard error of the mean. Results were analyzed using a two-tailed Student's t-test for unpaired data. For multiple comparisons analysis of variance was performed, followed by the Kruskal-Wallis test and two-sided Mann-Whitney U Test, where appropriate. Kaplan-Meier curves were constructed and statistical analysis of the survival curves was performed using the log-rank test. For histological analysis of the collagen content, scar thickness and infarct size, the raw data were aggregated for every animal and statistical analysis was performed as described above. $\mathrm{P}<0.05$ was considered to indicate a statistically significant difference. PAWS Statistics for Windows, version 18 (SPSS Inc., Chicago, IL, USA) was used.

\section{Results}

Survival rates. The number of animals that succumbed during the different study periods and those included into the final analysis are shown in Fig. 1. The majority of the animals that succumbed up to day 28 had a large MI and signs of severe heart failure in the autopsies (pneumonia, $n=2$ and hematothorax, $n=1)$. All the animals that succumbed within the treatment period of days 28-182 had a large MI, reaching from the base to the apex of the LV and comprising more than 
Table II. Body and organ weights six months after large myocardial infarction or sham-operation.

\begin{tabular}{lcrr}
\hline Parameter & Plac $(\mathrm{n}=27)$ & Tra $(\mathrm{n}=25)$ & ShO $(\mathrm{n}=18)$ \\
\hline BW $_{182}, \mathrm{~g}$ & $282.00 \pm 5.79$ & $268.32 \pm 6.91$ & $293.06 \pm 6.48$ \\
Weight gain, g & $52.21 \pm 7.60$ & $7.15 \pm 6.10$ & $67.07 \pm 5.73$ \\
LV, mg & $742.2 \pm 12.3^{\mathrm{b}}$ & $796.6 \pm 23.5^{\mathrm{b}}$ & $614.1 \pm 14.2$ \\
LVI, mg/g & $2.67 \pm 0.06^{\mathrm{b}}$ & $2.99 \pm 0.09^{\mathrm{b}, \mathrm{d}}$ & $2.09 \pm 0.06$ \\
RV, mg & $197.0 \pm 14.0^{\mathrm{c}}$ & $209.0 \pm 15.8^{\mathrm{b}}$ & $144.1 \pm 9.1$ \\
RVI, mg/g & $0.70 \pm 0.05^{\mathrm{c}}$ & $0.80 \pm 0.07^{\mathrm{b}}$ & $0.49 \pm 0.03$ \\
& & & \\
\hline
\end{tabular}

Data are presented as the mean \pm standard error of the mean. ${ }^{a}$ Weight gain is the difference of the body weight between day 182 and the time of surgery. ${ }^{b} \mathrm{P}<0.001$ vs. ShO, ${ }^{c} \mathrm{P}<0.05$ vs. ShO and ${ }^{\mathrm{d}} \mathrm{P}<0.05$ vs. Plac. Groups: Plac, large myocardial infarction/placebo; Tra, large myocardial infarction/tranilast; ShO, sham-operation/placebo. $\mathrm{BW}_{182}$, body weight at day $182 ; \mathrm{LV}$, Weight of the left ventricle; LVI, LV per body weight; $\mathrm{RV}$, weight of the right ventricle; RVI, RV per body weight.

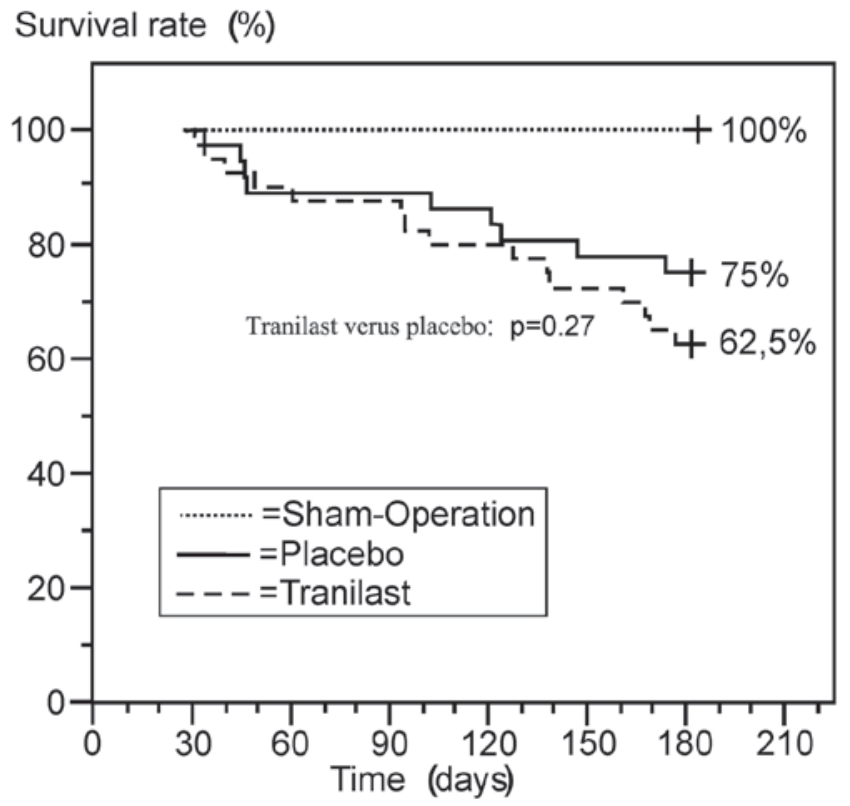

Figure 2. Kaplan-Meier analysis and survival rates six months after large myocardial infarction (tranilast, $\mathrm{n}=40$; placebo, $\mathrm{n}=36$; sham-operation, $\mathrm{n}=18$ ).

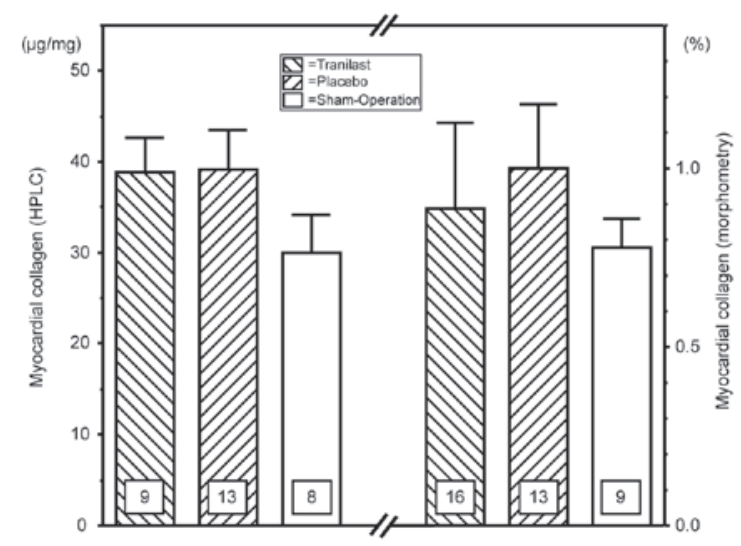

Figure 3. Intracardiac collagen content in the remote non-infarcted section of the left ventricle six months after large myocardial infarction. The collagen content was measured via HPLC or pixel-based analysis of stained cryosections on the left and right sides of the graph, respectively. Data are presented as the mean \pm standard error of the mean. HPLC, high-performance liquid chromatography. one-third of its circumference. No animal succumbed during the treatment period following the ShO.

At the end of the study period, 36 rats in the tranilast group, 36 rats in the placebo group and 18 rats following the $\mathrm{ShO}$ were sacrificed. Their organs and blood samples were obtained for analysis. There was no infarction of the right ventricle observed. None of the sham-operated rats had MI. In 11 rats of the tranilast group and in nine rats of the placebo group, the sizes of the MIs were too small to fit the aforementioned criteria. These animals were excluded from the Kaplan-Meier analysis and the weights of these lungs and hearts were analyzed separately. This led to a further reduction in the size of the groups, but enabled a comparison between the survivors and non-survivors. Finally, 40 animals that were treated with tranilast (15 succumbed and 25 survived) and 36 animals that were treated with placebo (nine succumbed and 27 survived) had a large MI, resulting in six-month survival rates of 62.5 and $75 \%$, respectively. The curves in the Kaplan-Meier analysis were similar (Fig. 2).

The body weights at day 182 tended to differ among the three study groups, with the lowest body weight in the tranilast group, without reaching statistical significance $(\mathrm{P}=0.051)$. Induction of large MI led to a significant hypertrophy of the LV and right ventricle. However, the weights of the LV in the tranilast group were higher than those in the placebo group and this combination of lower body weight and higher weight of the LV led to a significant difference of the left-ventricular-index [heart weight (mg)/body weight $(\mathrm{g})$ ], between the two groups in the statistical analysis (Table II).

Cardiac collagen content following large MI. The cardiac hypertrophy following large MI was accompanied by an increase of intracardiac collagen content in the remote non-infarcted part of the LV. This aspect was shown in the histological analysis, as well as in the measurement of the hydroxyproline by HPLC. However, the two methods revealed that the difference compared with the hearts following the $\mathrm{ShO}$ was not statistically significant. The levels of collagen content were not significantly different between the treatment groups following MI (Fig. 3).

A severe dilatation of the LV following large MI could be found in the analysis of the pressure volume curves of the 
Table III. Histological evaluation of the infarct size, dilatation of the LV and scar thickness following large myocardial infarction or sham-operation (pixel-based analysis of stained cryosections).

\begin{tabular}{lrrr}
\hline Parameter & Tra $(\mathrm{n}=16)$ & Plac $(\mathrm{n}=13)$ & ShO $(\mathrm{n}=9)$ \\
\hline Circumference LV, mm & $26.67 \pm 0.85$ & $28.32 \pm 0.96$ & $14.77 \pm 0.64^{\mathrm{a}}$ \\
Scar length, mm & $9.91 \pm 0.55$ & $10.66 \pm 0.46$ & $40.44 \pm 1.67$ \\
Scar, \% of circumference & $37.29 \pm 1.80$ & $0.750 \pm 0.038$ \\
Scar thickness, mm & $0.705 \pm 0.034$ & \\
\hline
\end{tabular}

Data are presented as the mean \pm standard error of the mean. ${ }^{a} \mathrm{P}<0.001$ vs. Tra and Plac. Groups: Plac, large myocardial infarction/placebo; Tra, large myocardial infarction/tranilast; ShO, sham-operation/placebo. LV, left ventricle.

Table IV. Body and organ weights and intracardiac collagen content in the remote non-infarcted part of the left ventricle obtained from the animals that succumbed during the treatment period following large myocardial infarction.

\begin{tabular}{lcc}
\hline Parameter & Plac $(\mathrm{n}=9)$ & Tra $(\mathrm{n}=15)$ \\
\hline Survival time, days & $170 \pm 26$ & $163 \pm 18$ \\
Collagen content, \% ${ }^{\mathrm{a}}$ & $0.98 \pm 0.13$ & $1.00 \pm 0.44$ \\
BWR & $1.15 \pm 0.13$ & $1.11 \pm 0.05$ \\
LGR & $5.72 \pm 0.37$ & $5.37 \pm 0.24$ \\
LVI, mg/g & $3.23 \pm 0.17$ & $3.13 \pm 0.14$ \\
RVI, mg/g & $1.36 \pm 0.21$ & $1.48 \pm 0.15$
\end{tabular}

Data are presented as the mean \pm standard error of the mean. ${ }^{\mathrm{a}} \mathrm{Col}-$ lagen content is measured as the ratio of collagen-stained area over total stained area in the cryosections. Groups: Plac, large myocardial infarction/placebo; Tra: large myocardial infarction/tranilast. BWR, ratio of body weight at time of mortality over that at the time of surgery; LGR, ratio of the wet over dry weight of the lungs; LVI, weight of left ventricle per body weight; RVI, weight of right ventricle per body weight.

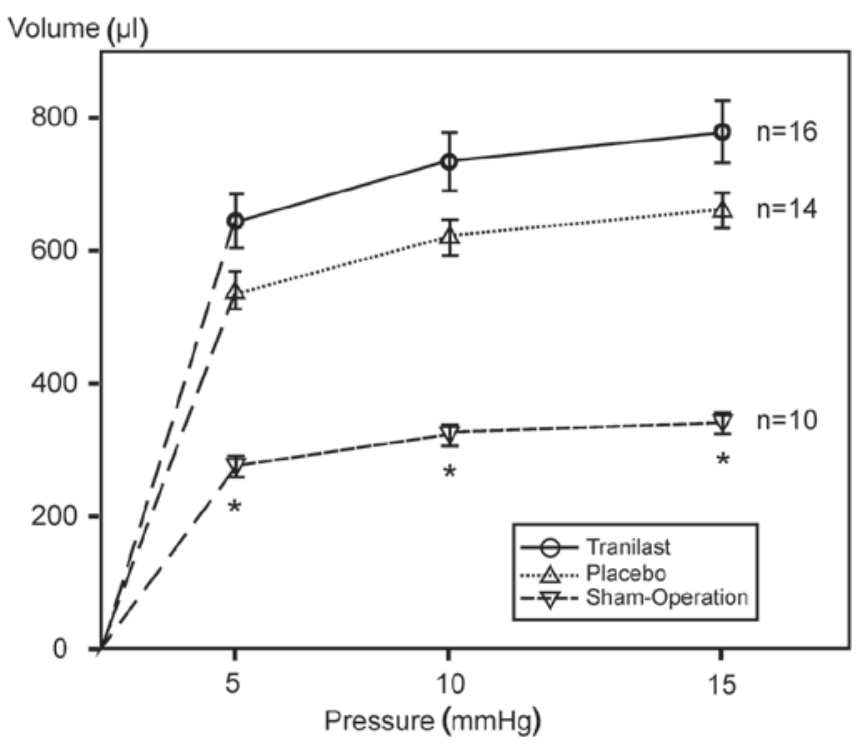

Figure 4. Pressure-volume association in the isolated left ventricle six months after large myocardial infarction or sham-operation. Data are presented as the mean \pm standard error of the mean. ${ }^{*} \mathrm{P}<0.001$ vs. tranilast and placebo.

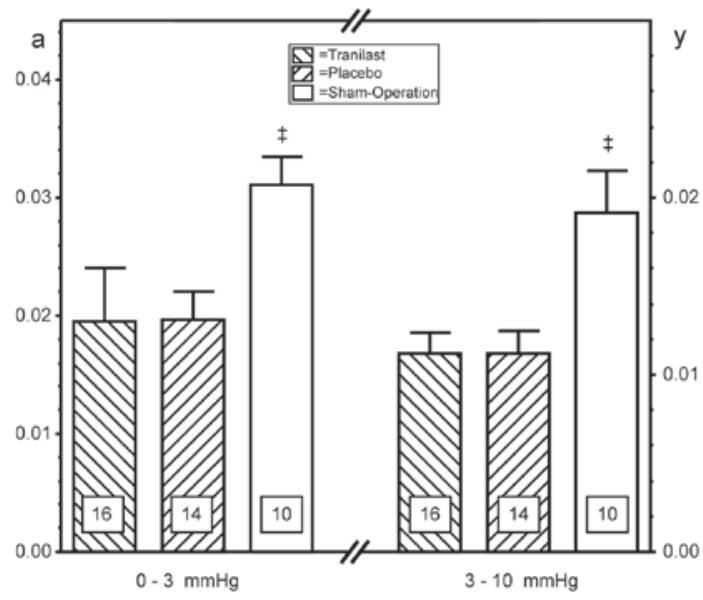

Figure 5. Calculation of the chamber stiffness variables. Analysis of resting pressure-volume curves of the isolated left ventricle, six months after large myocardial infarction or sham-operation. Left: 'a' derived by linear regression analysis $(y=a x+b)$ at the pressure range, 0-3 mmHg. Right: ' $d$ ' of the isolated left ventricle derived by exponential regression analysis $(\mathrm{y}=\mathrm{c} \mathrm{x} \mathrm{xd})$ of resting pressure-volume curves at the pressure range, 3-10 $\mathrm{mmHg}$. Data are presented as the mean \pm standard error of the mean. ${ }^{\ddagger} \mathrm{P}<0.05$ vs. tranilast and placebo.

isolated LV (Fig. 4), as well as by measuring the circumference of the LV in the histological analysis. The infarct size was the same in the two treatment groups. Treatment with tranilast did not lead to a difference in the scar extension or thickness (Table III).

Corresponding to the morphological changes following MI described above, a decrease of the chamber stiffness variables 'a' (pressure range, 0-3 mmHg) and 'd' (pressure range, $3-10 \mathrm{mmHg}$ ) was measured in the two treatment groups, without any significant difference between the treatment groups (Fig. 5).

Non-survivors. Following analysis of the organs from the animals that succumbed during the treatment period with large MI, no relevant difference between the treatment groups was found regarding the weight gain of the animals or the ventricular weight or the collagen content (histology) in the remote non-infarcted part of the LV (Table IV). Of note are the high weights of the ventricles compared with those of the animals that survived over the study period. This finding can be interpreted as a sign of an extensive cardiac hypertrophy, 
Table V. Body and organ weights, collagen content and results of the pressure volume analysis of the isolated left ventricle, six months after small myocardial infarction or sham-operation.

\begin{tabular}{lccc}
\hline Parameter & Plac $(\mathrm{n}=9)$ & Tra $(\mathrm{n}=11)$ & ShO $(\mathrm{n}=18)$ \\
\hline BWR & $1.44 \pm 0.03^{\mathrm{a}}$ & $1.24 \pm 0.05$ & $1.30 \pm 0.03$ \\
LGR & $5.51 \pm 0.27$ & $5.78 \pm 0.22$ & $5.51 \pm 0.24$ \\
$\mathrm{LVI}, \mathrm{mg} / \mathrm{g}$ & $2.31 \pm 0.21$ & $2.34 \pm 0.16$ & $2.09 \pm 0.06$ \\
RVI, mg/g & $0.50 \pm 0.05$ & $0.54 \pm 0.04$ & $0.49 \pm 0.03$ \\
$\mathrm{CSV}$ at $0-3 \mathrm{mmHg}$ & $0.022 \pm 0.003$ & $0.115 \pm 0.049$ & $0.031 \pm 0.002$ \\
CSV at 3-10 mmHg & $0.008 \pm 0.002$ & $0.018 \pm 0.003$ & $0.019 \pm 0.002$ \\
Volume 5 mmHg & $398.2 \pm 43.2$ & $326.7 \pm 40.7$ & $283.4 \pm 16.2$ \\
Volume $10 \mathrm{mmHg}$ & $483.7 \pm 35.9$ & $377.5 \pm 33.1$ & $323.2 \pm 15.3$ \\
Volume $15 \mathrm{mmHg}$ & $546.3 \pm 33.8$ & $411.6 \pm 28.1$ & $342.7 \pm 15.6$ \\
Collagen $\mathrm{HPLC}(\mu \mathrm{g} / \mathrm{mg})$ & $41.31 \pm 4.83(\mathrm{n}=5)$ & $39.98 \pm 5.87(\mathrm{n}=8)$ & $29.98 \pm 4.18(\mathrm{n}=5)$ \\
\hline
\end{tabular}

${ }^{\mathrm{a}} \mathrm{P}<0.05$ vs. Tra. Data are presented as the mean \pm standard error of the mean. Groups: Plac, large myocardial infarction/placebo; Tra, large myocardial infarction/tranilast; ShO, sham-operation/placebo. BWR, ratio of body weight at time of mortality over that at the time of surgery; LGR, ratio of the wet over dry weight of the lungs; LVI, weight of left ventricle per body weight; RVI, weight of right ventricle per body weight; CSV, chamber stiffness variable; HPLC, high-pressure liquid chromatography.

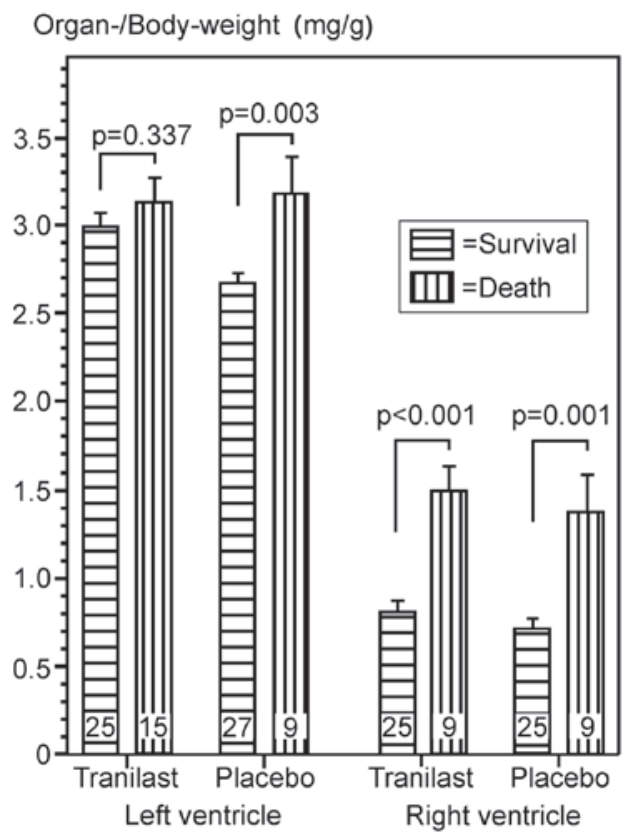

Figure 6. Weight of left and right ventricles per body weight in rats surviving the six month time period after large myocardial infarction, or succumbing in this time due to heart failure. Numbers in the bars show the group sizes (n). Data are presented as the mean \pm standard error of the mean.

which led to a progressive ventricular failure and thereby to the early mortality of these animals (Fig. 6).

Small MIs. Compared with the sham-operated hearts, the induction of small MIs led to a moderate increase of the weight, the intracardiac collagen content and a moderate dilatation of the LV, without reaching statistical significance. The ratios of the wet/dry weight of the lungs and the weights of the right ventricles were found at similar levels in all the groups. Chronic left ventricular failure induced following small MI was not severe enough to determine pulmonary edema and secondary right ventricular hypertrophy. Treatment with tranilast led to a lower weight gain compared with placebo. Regarding the other parameters, no statistically significant difference could be found between the treatment groups (Table V).

\section{Discussion}

Treatment with the anti-inflammatory and antifibrotic drug tranilast, initiated four weeks after the induction of a large MI in the rat, did not attenuate the cardiac remodeling in chronic ischemic heart failure. There was no effect on the six-month mortality rate. These findings indicate that extremely late tranilast therapy does not positively influence recovery and remodeling in this setting.

By contrast, animal models of salt hypertensive rats, spontaneously hypertensive rats, renovascular hypertension and experimental diabetic cardiomyopathy have been reported to positively influence by tranilast therapy (13). Cardiac interstitial fibrosis and remodeling have been shown to improve, however, similarly the pathogenic mechanisms, as well as the therapeutic interventions, involve the entire LV in their whole circumference of the per se viable myocardium. When treatment is successful, the recovery process also comprises the entire circumference of the LV. The process of cardiac remodeling in the setting of definite myocardial infarction is different and can be described as a response of the remote non-infarcted section of the LV to cell damage in another area of the heart. These processes involve changes in ventricular geometry and shape during systolic contraction. The pathological background consists of myocyte hypertrophy, apoptosis and interstitial fibrosis in the remote non-infarcted area. Several studies examined the effects of revascularization procedures in acute myocardial ischemia. Therapeutic interventions, such as revascularization procedures, started in the early phase lead to the salvage of myocardial tissue at 
risk and reverse local dysfunction in the ischemic area. When effective, reverse geometric remodeling is observed and this is associated with beneficial effects on the left ventricular function, not only in the ischemic but also in the remote part of the LV (23). In addition, coronary revascularization of patients with viable, also known as hibernating, myocardium in the infarcted zone positively influenced cardiac remodeling and prevented further major cardiac events, but these interventions were not effective, if the ventricles were severely dilated and the ejection fraction was too low (24). In patients undergoing coronary revascularization during or subsequent to an acute MI, the left ventricular end-systolic volume could be identified as the most important discriminator for the development of heart failure and mortality in patients following MI, irrespective of the revascularization status (25). Any therapeutic approach other than revascularization during the acute phase of the MI may prevent further damage in the remote non-infarcted area, but the dilatation and change of geometry of the LV due to necrotic tissue cannot be reversed. In the present experimental setting, large MIs with prominent LV dilatation were induced. Starting treatment with tranilast four weeks after the induction of the large MI was too late to induce reverse cardiac remodeling. At this time the severe dilatation of the LV that was found after six months may already have been completed. Recently, it has been described that tranilast intervention from $24 \mathrm{~h}$ post-MI exacerbated infarct expansion, but delayed the commencement of treatment to seven days post-MI impeded LV remodeling (19). Taken together with the present data, starting on day 7 appears to be the optimal timing.

The extremely late time point of treatment initiation had been chosen to prevent an interaction of tranilast with the wound healing processes in the infarcted area of the LV with the risk of the formation of left ventricular aneurysms. Interactions of this type are described for other anti-inflammatory drugs administered during the acute phase of MI $(26,27)$. During this maturation phase, the fibroblasts and vascular cells in the infarcted area undergo apoptosis. Cross-links are formed between the collagen bundles of the developing scar. After four weeks an organized assembly of collagen fibers in terms of scar tissue is found in the infarcted area of the LV (28). However, this appears not to be of relevance regarding the study endpoints.

Limitations of the present study include that it was performed in female Lewis rats. Compared with our previous results in male Lewis rats, lower levels of interstitial collagen were found measured in the two methods following $\mathrm{ShO}$, as well as subsequent to the induction of a large MI (29). Similar results were reported for the animal model of uni-nephrectomized DOCA plus salt hypertensive mice. While the ratio of the heart weight to the body weight after four weeks was significantly increased in male animals, this was not the case in female animals (30). Gender differences can also be found concerning the mRNA levels for $T G F-\beta_{1}$ (31). The different results in male rats cannot be excluded. Care was taken during the procedure of the present study to produce large MIs by ligating the left anterior descending coronary artery as proximally as possible. Every animal that succumbed during the study period was autopsied to ensure that the mortality was caused by a large MI and subsequent heart failure. At the end of the study period, every heart was examined and only rats with comparable large MIs were included in the analysis. The aim was to create comparable study groups. The major drawbacks of this strategy were the high mortality rate in the perioperative time (44\%) and an additional loss of animals at the end of the study period due to too small MIs (18\%). There was only a small number of hearts available for the analysis of cardiac remodeling at the end of the study period.

Treatment with the anti-inflammatory and antifibrotic drug, tranilast, started four weeks after the induction of a large MI in the rat, does not attenuate or positively influence remodeling in chronic ischemic heart failure and survival rate. Further studies are required to explore the effects of tranilast on cardiac myocytes post-MI in more detail.

\section{Acknowledgements}

The authors would like to thank Mrs. Martina Voigt for her excellent technical assistance.

\section{References}

1. Azuma H, Banno K and Yoshimura T: Pharmacological properties of $\mathrm{N}-\left(3^{\prime}, 4^{\prime}-\right.$ dimethoxycinnamoyl) anthranilic acid (N-5'), a new anti-atopic agent. Br J Pharmacol 58: 483-488, 1976.

2. Taniguchi S, Yorifuji T and Hamada T: Treatment of linear localized scleroderma with the anti-allergic drug, tranilast. Clin Exp Dermatol 19: 391-393, 1994.

3. Yamada H,Ide A, Sugiura M and Tajima S: Treatment of cutaneous sarcoidosis with tranilast. J Dermatol 22: 149-152, 1995.

4. Yamada H, Tajima S, Nishikawa T, Murad S and Pinnell SR: Tranilast, a selective inhibitor of collagen synthesis in human skin fibroblasts. J Biochem 116: 892-897, 1994.

5. Suzawa H, Kikuchi S, Ichikawa K and Koda A: Inhibitory action of tranilast, an anti-allergic drug, on the release of cytokines and PGE2 from human monocytes-macrophages. Jpn J Pharmacol 60: 85-90, 1992.

6. Adachi S, Maruyama T, Kondo T, Todoroki T and Fukao K: The prevention of postoperative intraperitoneal adhesions by tranilast: N-(3',4'-dimethoxycinnamoyl)anthranilic acid. Surg Today 29: 51-54, 1999.

7. Nakamura K, Irie H, Inoue M, Mitani H, Sunami $H$ and Sano S: Factors affecting hypertrophic scar development in median sternotomy incisions for congenital cardiac surgery. J Am Coll Surg 185: 218-223, 1997.

8. Watanabe S, Matsuda A, Suzuki Y, et al: Inhibitory mechanism of tranilast in human coronary artery smooth muscle cells proliferation, due to blockade of PDGF-BB-receptors. Br J Pharmacol 130: 307-314, 2000.

9. Takahashi A, Taniguchi T, Ishikawa $\mathrm{Y}$ and Yokoyama M: Tranilast inhibits vascular smooth muscle cell growth and intimal hyperplasia by induction of p21(waf1/cip1/sdi1) and p53. Circ Res 84: 543-550, 1999.

10. Tamai H1, Katoh O, Suzuki S, et al: Impact of tranilast on restenosis after coronary angioplasty: tranilast restenosis following angioplasty trial (TREAT). Am Heart J 138: 968-75, 1999.

11. Tamai H, Katoh K, Yamaguchi T, et al: The impact of tranilast on restenosis after coronary angioplasty: the Second Tranilast Restenosis Following Angioplasty Trial (TREAT-2). Am Heart J 143: 506-513, 2002.

12. Holmes DR Jr, Savage M, LaBlanche JM, et al: Results of Prevention of REStenosis with Tranilast and its Outcomes (PRESTO) trial. Circulation 106: 1243-1250, 2002.

13. Umemura K, Kikuchi S, Suzuki Y and Nakashima M: Inhibitory effect of tranilast on hypertrophic collagen production in the spontaneously hypertensive rat heart. Jpn J Pharmacol 78: 161-167, 1998.

14. Kagitani S, Ueno H, Hirade S, Takahashi T, Takata M and Inoue $\mathrm{H}$ : Tranilast attenuates myocardial fibrosis in association with suppression of monocyte/macrophage infiltration in DOCA/salt hypertensive rats. J Hypertens 22: 1007-1015, 2004. 
15. Bujak M and Frangogiannis NG: The role of TGF-beta signaling in myocardial infarction and cardiac remodeling. Cardiovasc Res 74: 184-195, 2007.

16. Dewald O, Ren G, Duerr GD, et al: Of mice and dogs: species-specific differences in the inflammatory response following myocardial infarction. Am J Pathol 164: 665-677, 2004

17. Cohn JN, Ferrari R and Sharpe N: Cardiac remodeling - concepts and clinical implications: a consensus paper from an international forum on cardiac remodeling. Behalf of an International Forum on Cardiac Remodeling. J Am Coll Cardiol 35: 569-582, 2000.

18. Ikeuchi M, Tsutsui H, Shiomi T, et al: Inhibition of TGF-beta signaling exacerbates early cardiac dysfunction but prevents late remodeling after infarction. Cardiovasc Res 64: 526-535, 2004.

19. See F, Watanabe M, Kompa AR, et al: Early and delayed tranilast treatment reduces pathological fibrosis following myocardial infarction. Heart Lung Circ: Sept 14, 2012 (Epub ahead of print)

20. Jung C, Gonon AT, Sjöquist PO, Lundberg JO and Pernow J: Arginase inhibition mediates cardioprotection during ischaemia-reperfusion. Cardiovasc Res 85: 147-154, 2010.

21. Pfeffer MA, Pfeffer JM, Fishbein MC, Fletcher PJ, Spadaro J, Kloner RA, Braunwald E. Myocardial infarct size and ventricular function in rats. Circ Res 44: 503-512, 1979.

22. Fletcher PJ, Pfeffer JM, Pfeffer MA and Braunwald E: Left ventricular diastolic pressure-volume relations in rats with healed myocardial infarction. Effects on systolic function. Circ Res 49: 618-626, 1981.

23. Carluccio E, Biagioli P, Alunni G, et al: Patients with hibernating myocardium show altered left ventricular volumes and shape, which revert after revascularization: evidence that dyssynergy might directly induce cardiac remodeling. J Am Coll Cardiol 47 969-977, 2006.
24. Bax JJ, Schinkel AF, Boersma E, et al: Extensive left ventricular remodeling does not allow viable myocardium to improve in left ventricular ejection fraction after revascularization and is associated with worse long-term prognosis. Circulation 110: II18-II22, 2004.

25. Senior R, Lahiri A and Kaul S: Effect of revascularization on left ventricular remodeling in patients with heart failure from severe chronic ischemic left ventricular dysfunction. Am J Cardiol 88: 624-629, 2001.

26. Brown EJ Jr, Kloner RA, Schoen FJ, Hammerman H, Hale S and Braunwald E: Scar thinning due to ibuprofen administration after experimental myocardial infarction. Am J Cardiol 51: 877-883, 1983

27. Bulkley BH and Roberts WC: Steroid therapy during acute myocardial infarction. A cause of delayed healing and of ventricular aneurysm. Am J Med 56: 244-250, 1974.

28. Weber KT, Sun Y and Cleutjens JP: Structural remodeling of the infarcted rat heart. EXS 76: 489-499, 1996.

29. Betge S, Lutz K, Roskos M and Figulla HR: Oral treatment with probucol in a pharmacological dose has no beneficial effects on mortality in chronic ischemic heart failure after large myocardial infarction in rats. Eur J Pharmacol 558: 119-127, 2007.

30. Karatas A, Hegner B, de Windt LJ, et al: Deoxycorticosterone acetate-salt mice exhibit blood pressure-independent sexual dimorphism. Hypertension 51: 1177-1183, 2008.

31. Tyagi P, Tyagi V, Yoshimura N, et al: Gender-based reciprocal expression of transforming growth factor-betal and the inducible nitric oxide synthase in a rat model of cyclophosphamide-induced cystitis. J Inflamm (Lond) 6: 23, 2009. 